\title{
Efficacy of Combined Omalizumab with Cat allergen Immunotherapy in Cat induced Allergic Rhino-conjunctivitis \& intermittent Asthma-Case Report
}

\section{Kathuria PC*, Manisha Rai}

BLK Super Specialty Hospital \& National Allergy Centre, New Delhi, India

*Corresponding author: Kathuria PC, BLK Super Specialty Hospital \& National Allergy Centre, New Delhi, India.

Citation: Kathuria PC, Rai M (2021) Efficacy of Combined Omalizumab with Cat allergen Immunotherapy in Cat induced Allergic Rhino-conjunctivitis \& intermittent Asthma-Case Report. Arch Surg Clin Case Rep 4: 159. DOI: 10.29011/2689-0526.100159

Received Date: 30 November, 2021; Accepted Date: 03 December, 2021; Published Date: 08 December, 2021

\section{Abstract}

The prevalence of allergy to furry animals has been increasing and allergy to cats is a major risk factor for the development of Rhinitis \& Asthma, poor lung function, increased airway inflammation, airway hyperresponsiveness and re-modelling. We report the case of 42-year-old male with cat-induced allergic rhino-conjunctivitis and intermittent asthma, who achieved remission in six months after initiating avoidance measures along with combined Omalizumab (Anti-IgE) and Subcutaneous Allergen Immunotherapy (SCIT). The patient was followed-up for 3 years without any recurrence of symptoms of allergic rhino-conjunctivitis and intermittent asthma. We speculate that SCIT combined with Omalizumab in mono-sensitized patients can induce successful early and faster remission of disease.

Keywords: CatAllergy; Cat Hair Extract; Fel d 1; Omalizumab; Anti IgE; Subcutaneous Allergen Immunotherapy (SCIT)

\section{Introduction}

Furry animals are one of the major sources of indoor allergens and are considered as risk factors for allergic diseases. These allergens are very stable and persist in house dust for 6 months. Cats and dogs are the most common among household pets worldwide. Allergy to dogs and cats has long been considered a major risk factor in the development of allergic rhinoconjunctivitis and intermittent asthma. Sensitization to dog hair was found to be $11.8 \%$ and cat hair $8.1 \%$ in the developed region compared to $7.1 \%$ and $8.7 \%$ respectively in the developing region of the pearl delta in Guangdong China [1,2].

Interestingly, both dog and cat allergen exposure are ubiquitous even in public places, can be observed in individuals who live in a house where cats are not present. Animal allergens are easily transported by clothes. The most frequent allergy symptoms among cat owners with a cat allergy during contact with their cats were rhinitis $(80 \%)$, followed by conjunctivitis $(73.3 \%)$, cutaneous symptoms (33.3\%) and lower respiratory symptoms (13.3\%) [3].
Dog owners with a Dog allergy suffered from cutaneous symptoms more frequently than cat owners with a Cat allergy $(55.3 \%$ vs $33.3 \%, p<0.01)$, especially in terms of urticaria $(35.9 \%$ vs $15.6 \% \mathrm{p}<0.01)$. dog owners with a dog allergy also experienced lower respiratory symptoms more frequently than did cat owners with a cat allergy $(33.3 \%$ vs $13.3 \% \mathrm{p}<0.05)$, especially cough $(23.3 \%$ vs $6.7 \% \mathrm{p}<0.05$ [3].

Lipocalins constitute the most important allergenic protein family. Most of which are major allergens Fel d 4, Can $\mathrm{f} 1$, Can f 6 and Equ c1. Fel d 4 are lipocalins, are transporter for small hydrophobic molecules such as lipids, steroid hormones, bilins and retinoids. Allergens from this protein family include b-lactoglobulin, mammalian dander allergens and cytoplasmic fatty acid binding proteins [4]. They are abundant in saliva and dander. Fel d 1 , the major cat allergen is about $38 \mathrm{kDa}$ in size. It is a thermostable, uteroglobin expressed in salivary glands and sebaceous glands on skin surface and fur. $\mathrm{IgE}$ to Fel $\mathrm{d} 1$ is equally good as IgE to cat extract in predicting cat allergy. It is said that concentration of 1-8ug/gm powder of Can $\mathrm{f} 1$ and Fel d 1 of 2-20 nanograms per cubic meter in the air seem to be associated with higher risk of developing sensitization to dogs and cats as well as 
Citation: Kathuria PC, Rai M (2021) Efficacy of Combined Omalizumab with Cat allergen Immunotherapy in Cat induced Allergic Rhino-conjunctivitis \& intermittent Asthma-Case Report. Arch Surg Clin Case Rep 4: 159. DOI: 10.29011/2689-0526.100159

causing symptoms of allergic diseases [5].

As patients who are allergic to dog and cat are rarely willing to give up their pets, so pharmacotherapy and AIT may become the main methods of treatment. Allergen specific high dose SCIT has been shown to be beneficial in cat allergic patient with allergic rhino-conjunctivitis and intermittent asthma, whereas the data for dog-allergic patients are less convincing $[3,6,7]$.

\section{Case Presentation}

42 years old male, on treatment of hypothyroidism, presented with history of difficulty in breathing associated with itching in the eyes within half an hour of contact with a cat. Symptoms developed 6 months back when the patient bought a domestic cat home. His past medical history was insignificant for asthma or any of atopic diseases. Allergen specific IgE against cat was 18.4 $\mathrm{kUA} / \mathrm{L}$, and negative against dog by ImmunoCAP assay ${ }^{\circledR}$. Skin prick test (SPT) was $5 \mathrm{~mm}$ for histamine, $10 \mathrm{~mm}$ for commercial extract of cat dander and negative for pollens, fungus and other animal dander's.

The patient was diagnosed to have rhino-conjunctivitis and intermittent asthma on exposure to Cat dander (mono- sensitized), in spite of symptomatic treatment (Intra-nasal steroids, Leukotriene antagonist, Inhaled Corticosteroids, Long-acting beta Agonist). His lung function (FEV1-88\%, FEV1/FVC- 92.5\%) was within normal limit (no airway re-modelling) when the patient was away from home environment. Our patient was treated with strict avoidance measures and combined SCIT and Omalizumab. He got remission in six months and was followed-up for 3 years after discontinuation of SCIT.

A subcutaneous immunotherapy protocol was designed with a built-up phase of 6-7 weeks. It was difficult to achieve the maintenance dose of SCIT, 1000 BAU per $0.5 \mathrm{ml}$ of Standardized cat hair Allergenic extract, because of persistent symptoms and poor response to conventional and supportive therapy of Allergic Rhino-conjunctivitis and intermittent Asthma. We planned pretreatment with Omalizumab $(150 \mathrm{mg}) 15$ days before the Cat hair extract SCIT with a modified cluster schedule (Table 1) at interval of 10-15 days, maintenance dose of 1000 BAU was achieved in 4 visits, (6 to 7 weeks). Omalizumab was administered for 6 months in our patient. He had no systemic reaction during immunotherapy. A large local allergic reaction was noted at injection site during the built-up phase. He was evaluated for efficacy in the first year and then at three years after discontinuation of SCIT.

\begin{tabular}{|c|c|c|c|c|c|c|}
\hline \multicolumn{7}{|c|}{ Inj Omalizumab (150mg) X 15 days before AIT followed by once in a month + AIT for 6 months } \\
\hline No. of Visits & $1^{\text {st }}$ Visit & $2^{\text {nd }}$ Visit & $3^{\text {rd }}$ Visit & $4^{\text {th }}$ Visit & $5^{\text {th }}$ Visit & $6^{\text {th }}$ Visit \\
\hline $\begin{array}{l}\text { Cluster Dose AIT (1000 BAU per } \\
0.5 \mathrm{ml} \text { per MD) } \\
\text { Combined with Inj Omalizumab } \\
(\mathbf{1 5 0 m g})^{*}\end{array}$ & $\begin{array}{l}0.05 / 0.05 \mathrm{ml} \\
\text { ( } 60 \mathrm{mts} \text { interval } \\
(200 \mathrm{BAU})\end{array}$ & $\begin{array}{l}0.1 / 0.1 \mathrm{ml} \\
@ \quad 60 \mathrm{mts} \\
\text { interval } \\
(400 \mathrm{BAU})\end{array}$ & $\begin{array}{l}0.2 / 0.2 \mathrm{ml} \\
@ \quad 60 \mathrm{mts} \\
\text { interval } \\
(800 \mathrm{BAU})\end{array}$ & $\begin{array}{l}0.3 / 0.2 \mathrm{ml} \\
\text { @ } 60 \mathrm{mts} \\
\text { interval } \\
(1000 \mathrm{BAU})\end{array}$ & $\begin{array}{l}0.5 \mathrm{ml} \\
\left(\begin{array}{llll}1 & 0 & 0 & 0 \\
\text { BAU }\end{array}\right)\end{array}$ & (1000 BAU) \\
\hline Cluster Dose frequency & First day & 10-12 days & 10-12 days & 15-20 days & $\begin{array}{l}20-30 \\
\text { days }\end{array}$ & $\begin{array}{l}\text { Every } \\
\text { weeks }\end{array}$ \\
\hline
\end{tabular}

Table 1: Schedule and duration of Combined standardized Cat hair Subcutaneous Cluster Immunotherapy (Cat SCIT) $(1 \mathrm{ml}=10,000$ BAU) along with Inj Omalizumab therapy $150 \mathrm{mg}$ per month.

\section{Discussion}

Our patient with allergic rhino-conjunctivitis and intermittent asthma due to cat allergy was treated with strict avoidance measures and combined SCIT and Omalizumab. He got remission in 6 months and was followed up for 3 years after discontinuation of SCIT. Allergic rhino-conjunctivitis and intermittent asthma due to cat allergy is an immunological disease with a predominance of Th2 lymphocytic inflammation. The diagnosis of cat allergy is based on a consistent medical history and physical examination and is confirmed by a positive skin test or specific IgE. Our patient was monosensitized to cat allergen with signs and symptoms of allergic rhino-conjunctivitis and intermittent asthma. Clinical allergists are commonly faced with cat owners who adamantly oppose to eliminating cat from their home environment but our patient took the initiative and a firm decision to avoid exposure to the cat was taken. It was well explained that presence of cat in their home will complicate management of allergic rhinitis and intermittent asthma. Moreover, the preventive measures including cat washing, keeping cat outside of bedrooms and the utilization of air filtration may be of little to no benefit, whereas persistent exposure will lead to progressive inflammation and re-modelling of airways [8-11].

Specific Allergen Immunotherapy is the only therapeutic method with a disease modifying, positive impact on natural course 
Citation: Kathuria PC, Rai M (2021) Efficacy of Combined Omalizumab with Cat allergen Immunotherapy in Cat induced Allergic Rhino-conjunctivitis \& intermittent Asthma-Case Report. Arch Surg Clin Case Rep 4: 159. DOI: 10.29011/2689-0526.100159

of allergic disease which persist even after the treatment has been stopped, conceived over 100 years back, it entails the application of gradually increasing doses of causative allergen. The approach has been shown to be effective in inducing immunological and clinical tolerance in the management of asthma, allergic rhinitis and venom allergies. The immunological mechanism of induction of tolerance involves rapid desensitization of mast cells and basophils, induction of $\mathrm{T}$ and $\mathrm{B}$ cells, blocking $\operatorname{IgG}$ antibody induction and a late-phase response suppression. Despite its efficacy carries a risk for inducing adverse reactions, which has been a critical issue.

The monoclonal anti-IgE antibody Omalizumab (Xolair, Novartis Pharma AB) binds IgE at the same site as these antibodies bind FceRI and FceRII on inflammatory cells. The bound IgE cannot bind to basophils thus attenuating degranulation and allergic symptoms. Omalizumab has proven efficacy in asthma trials by reducing inhaled corticosteroid as well as rescue medication usage. Similar benefits were seen when used in the management of seasonal allergic rhinitis (SAR) and perennial allergic rhinitis. Benefits were observed when combined with immunotherapy in SAR. A double blind randomized placebo-controlled trial was conducted to evaluate the efficacy of Omalizumab with specific immunotherapy (SIT) in children and adolescents with documented grass and birch pollen IgE and a history of SAR induced by both these allergens. A 24-week regimen of SIT with Omalizumab or placebo was initiated at week 12 . A $48 \%$ reduced median symptom load score was seen in the omalizumab arms as compared to their placebo arms [12].

In our patient of Cat induced Allergic Rhino-conjunctivitis $\&$ intermittent Asthma with normal lung function (Table 2) SCIT with Omalizumab has shown marked improvement in signs and symptoms (functional status returned to normal) and could achieve the maintenance dose faster in 6-7 weeks without any adverse effects. The Patient stopped AIT after 6 months and has been symptom-free for 3 years. He was protected from deleterious effects of inflammation and adverse outcome of irreversible airway remodelling. Our case points towards strong anti-inflammatory effect of SCIT with high and safe dose of Cat hair extract combined with Anti IgE Omalizumab in 6-7 weeks. The authors have published the efficacy of Cockroach Cluster Immunotherapy with Anti-IgE (Omalizumab) compared to conventional immunotherapy [13]. We propose that combining both can induce early tolerance to allergen extract with maximum dose and decrease the duration of allergen immunotherapy.

\begin{tabular}{|l|l|l|}
\hline & $\mathbf{2 0 1 9}$ & $\mathbf{2 0 2 1}$ \\
\hline Total IgE & $88.2 \mathrm{IU} / \mathrm{mL}$ & $72 \mathrm{IU} / \mathrm{ml}$ \\
\hline Specific IgE-Cat dander & $18.4 \mathrm{kUA} / 1$ & $5 \mathrm{kUA} / 1$ \\
\hline & & \\
$\begin{array}{l}\text { SPT- } \\
\text { Histamine }\end{array}$ & $5 \mathrm{~mm}$ & 0 \\
Cat epithelia & & $3 \mathrm{~mm}$ \\
& & 0 \\
& $10 \mathrm{~mm}$ & $3 \mathrm{~mm}$ \\
\hline FEV1 & & \\
\hline FEV1/FVC & $88 \%$ & $90 \%$ \\
\hline
\end{tabular}

Table 2: Clinical characteristics of the patient.

\section{Conclusion}

We hypothesize that pharmacotherapy is an option for a mild disease but SCIT and omalizumab ensures an effective and longlasting treatment in moderate to severe cases of allergic rhinoconjunctivitis and intermittent asthma. Avoidance measures and combined Cat hair extract SCIT with Omalizumab was successful in remission of the disease. Combined therapy is more effective than conventional AIT; it not only reduces the duration but also decreases the allergenicity of cat hair extract. However, we believe that there is still work to be carried out for the combination therapy of SCIT with Anti-IgE Omalizumab to prevent the onset of chronic inflammation and airway re-modelling.

\section{References}

1. Chan SK, Leung DYM, (2018). Dog and Cat Allergies: Current State of Diagnostic Approaches and Challenges. Allergy Asthma Immunol Res. 10: 97-105.

2. Qiu Q, Lu C, Han H, Chen S, (2013). Clinical survey and analysis of allergic rhinitis patients' allergens in Guangdong developed and developing regions. Lin Chung Er Bi Yan Hou Tou Jing Wai Ke Za Zhi 2013; 27: 928-31.

3. Yang MS, Lee SP, Kwon YJ, Lee SM, (2018). Dog and Cat Allergies and Allergen Avoidance Measures in Korean Adult Pet Owners Who Participated in a Pet Exhibition. Allergy Asthma Immunol Res. 2018; 10: 155-164. 
Citation: Kathuria PC, Rai M (2021) Efficacy of Combined Omalizumab with Cat allergen Immunotherapy in Cat induced Allergic Rhino-conjunctivitis \& intermittent Asthma-Case Report. Arch Surg Clin Case Rep 4: 159. DOI: 10.29011/2689-0526.100159

4. Satyaraj E, Wedner HJ, Bousquet J,(2019). Keep the cat, change the care pathway: A transformational approach to managing Fel $d$ 1, the major cat allergen. Allergy. 74(Suppl 107): 5-17.

5. Virtanen T, (2018). Immunotherapy for pet allergies. Hum Vaccin Immunother. 14: 807-814.

6. Ling M, Long AA, (2010). Pet dander and difficult-to-control asthma: therapeutic options. Allergy Asthma Proc. 31:385-91.

7. Dávila I, Domínguez-Ortega J, Navarro-Pulido A, Alonso A, AntolínAmerigo D, et al (2018). Consensus document on dog and cat allergy. Allergy. 73: 1206-1222.

8. Massanari M, Kianifard F, Zeldin RK, Geba GP (2009). Efficacy of omalizumab in cat-allergic patients with moderate-to-severe persistent asthma. Allergy Asthma Proc. 30: 534-9.

9. R. A. Wood, M. D. Chapman, N. F. Adkinson Jr., and P. A. Eggleston, (1989). "The effect of cat removal on allergen content in householddust samples," Journal of Allergy and Clinical Immunology. 83: 730734 .
10. C. Nageotte, M. Park, S. Havstad, E. Zoratti, and D. Ownby, (2006). "Duration of airborne Fel d 1 reduction after cat washing," Journal of Allergy and Clinical Immunology. 118: 521-522.

11. R. A. Wood, E. F. Johnson, M. L. Van Natta, Pei Hua Chen, and P. A. Eggleston, (1998). "A placebo-controlled trial of a HEPA air cleaner in the treatment of cat allergy," American Journal of Respiratory and Critical Care Medicine. 158: 115- 120.

12. Massanari M, Kianifard F, Zeldin RK, Geba GP, (2009). Efficacy of omalizumab in cat-allergic patients with moderate-to-severe persistent asthma. Allergy Asthma Proc. 30: 534-9.

13. Kathuria PC, Rai M (2020). Cockroach Cluster Immunotherapy with Anti-IgE (Omalizumab) versus Conventional Immunotherapy or AntiIgE (Omalizumab) alone in Five Cases of Allergic Rhinitis with Asthma. JSM Allergy Asthma 4: 1025. 